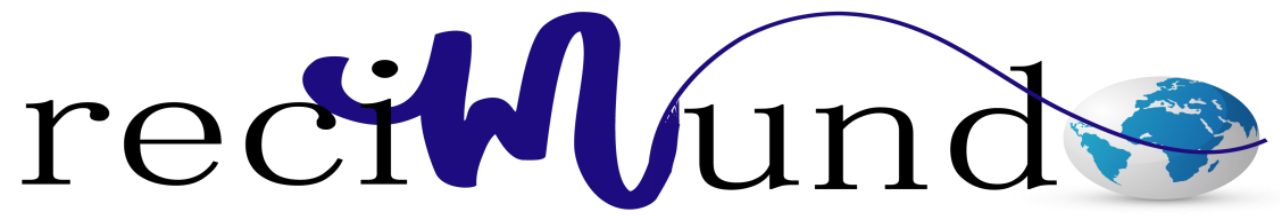

Revista Científica Mundo de la Investigación y el Conocimiento

Johanna Georgina Vidal Plúas a; Walter Alejandro Patiño Zambrano b; Luisana

Belen Solorzano López ${ }^{\text {c}}$; Steven Javier Berruz Alvarado ${ }^{\text {d }}$

Factores causales de hipertensión arterial en mujeres durante la gestación

Revista Científica Mundo de la Investigación y el Conocimiento. Vol. 2 núm.2, mayo, ISSN: 2588-073X, 2018, pp. 640-649

DOI: 10.26820/recimundo/2.(2).2018.640-649

Editorial Saberes del Conocimiento

Recibido: 05/12/2017

Aceptado: $15 / 04 / 2018$

a. Médico.

b. Médico; alejo8585@ hotmail.com

c. Hospital General de Chone Napoleón Dávila Córdova; luisana.solorzano@ hospitalgeneralchone.gob.ec

d. Universidad de Guayaquil Carrera de Medicina Estudiante de Medicina noveno Semestre; 


\section{Factores causales de hipertensión arterial en mujeres durante la gestación}

Vol. 2, núm. 2., (2018)

Johanna Georgina Vidal Plúas; Walter Alejandro Patiño Zambrano; Luisana Belen Solorzano López; Steven Javier Berruz Alvarado

\section{RESUMEN}

En el siguiente trabajo de investigación se realizó una investigación en referencia a los factores causales de la hipertensión arterial en las mujeres durante la gestación. Hay que destacar que la hipertensión arterial (HTA) es la complicación médica más frecuente durante el embarazo, además de representar la principal causa de mortalidad materna en todo el mundo. Durante la gestación se ha determinado que existen cinco tipos de alteraciones hipertensivas del embarazo: hipertensión crónica, hipertensión gestacional, la preeclampsia, preeclampsia sobreimpuesta en hipertensión crónica y la eclampsia. Se pudo obtener como resultado que en los pacientes con hipertensión arterial crónica se ha encontrado un aumento del riesgo de mortalidad perinatal, preeclampsia sobreagregada, parto prematuro, $\mathrm{RN}$ pequeño para la edad gestacional y cesárea. Debido al peligro de la preeclampsia se pudo concluir que sólo su diagnóstico precoz permitirá instaurar un tratamiento oportuno para lograr minimizar el alto riesgo de morbimortalidad, tanto materno como perinatal. También se pudo determinar entre las recomendaciones tener hábitos de vida saludable, el monitoreo estricto de presión arterial durante la consulta prenatal y programar con sus médicos de familia revisiones.

Palabras claves: Hipertensión arterial, embarazo, preeclampsia, cesárea y vida saludable. 


\title{
Factores causales de hipertensión arterial en mujeres durante la gestación
}

Vol. 2, núm. 2., (2018)

Johanna Georgina Vidal Plúas; Walter Alejandro Patiño Zambrano; Luisana Belen Solorzano López; Steven Javier Berruz Alvarado

\begin{abstract}
In the following research work an investigation was carried out in reference to the causal factors of hypertension in women during pregnancy. It should be noted that high blood pressure (HBP) is the most frequent medical complication during pregnancy, in addition to representing the main cause of maternal mortality worldwide. During pregnancy it has been determined that there are five types of hypertensive alterations of pregnancy: chronic hypertension, gestational hypertension, preeclampsia, preeclampsia superimposed in chronic hypertension and eclampsia. It was possible to obtain as a result that in patients with chronic arterial hypertension an increased risk of perinatal mortality, preaggregated preeclampsia, premature birth, small $\mathrm{RN}$ for gestational age and cesarean section has been found. Due to the danger of preeclampsia, it could be concluded that only early diagnosis will allow timely treatment to minimize the high risk of morbidity and mortality, both maternal and perinatal. It was also possible to determine among the recommendations to have healthy living habits, the strict monitoring of blood pressure during the prenatal consultation and to schedule reviews with their family doctors.
\end{abstract}

Key words: arterial hypertension, pregnancy, preeclampsia, caesarean section and healthy life. 


\section{Factores causales de hipertensión arterial en mujeres durante la gestación}

Vol. 2, núm. 2., (2018)

Johanna Georgina Vidal Plúas; Walter Alejandro Patiño Zambrano; Luisana Belen Solorzano López; Steven Javier Berruz Alvarado

\section{Introducción.}

La hipertensión arterial (HTA) es la complicación médica más frecuente durante el embarazo. Se presenta en el $8-10 \%$ de todas las gestaciones y es la principal causa de mortalidad materna en el mundo. La importancia de su estudio reside en que una atención médica prenatal adecuada puede prevenir o atenuar las alteraciones hipertensivas de la gestación, además la HTA en el embarazo es un marcador precoz tanto de HTA esencial como de enfermedad cardiovascular y renal futuras (Marin, Gorostidiz, \& Álvarez-Navascués, 2011).

La definición de HTA más aceptada es la misma que la admitida fuera de la gestación: presión arterial sistólica (PAS) mayor de $140 \mathrm{mmHg}$ y/o PA diastólica (PAD) mayor de 90 mmHg en dos o más ocasiones consecutivas y separadas por un período de cuatro-seis horas. La HTA durante la gestación se considera grave si la PAS es mayor de 160-170 mmHg y/o la PAD es mayor de 110 mmHg. (Marin, Gorostidiz, \& Álvarez-Navascués, 2011).

De acuerdo con la clasificación del National Blood Pressure Education Working Group 2000, existen 5 clases de alteraciones hipertensivas del embarazo, las cuales son: Hipertensión crónica, consiste en la presión arterial igual o mayor a 140/90 mmHg antes del embarazo o diagnosticada antes de la semana 20 de gestación. Hipertensión gestacional, dícese de la presión arterial igual o mayor a 140/90 $\mathrm{mmHg}$ sin proteinuria, que se desarrolla en etapas tardías del embarazo. Generalmente se resuelve en las primeras 12 semanas posparto. Si la hipertensión persiste después de este periodo, se realiza el diagnóstico de hipertensión crónica, enmascarada al principio del embarazo, por la disminución fisiológica de la presión arterial. Algunas mujeres que presentan hipertensión gestacional, desarrollan preeclampsia conforme progresa el 


\section{Factores causales de hipertensión arterial en mujeres durante la gestación}

Vol. 2, núm. 2., (2018)

Johanna Georgina Vidal Plúas; Walter Alejandro Patiño Zambrano; Luisana Belen Solorzano López; Steven Javier Berruz Alvarado

embarazo, especialmente si la hipertensión se presenta antes de las 30 semanas de gestación. (Noriega, Arias, \& García, 2005)

El tercer caso es la preeclampsia: presión arterial igual o mayor a 140/ $90 \mathrm{mmHg}$ asociada a proteinuria igual o mayor a $300 \mathrm{mg}$ en orina de 24 horas, después de las 20 semanas de gestación. La otra clase es la preeclampsia sobreimpuesta en hipertensión crónica, que se determina como la presencia de proteinuria igual o mayor a $300 \mathrm{mg}$ en orina de 24 horas en mujeres cuya presión arterial es igual o mayor a 140/90 $\mathrm{mmHg}$, antes del embarazo o diagnosticada antes de la semana 20 de gestación, pero que no presentaban proteinuria previamente. Por último, la eclampsia, que se presenta como la presencia de convulsiones en mujeres que cursen con preeclampsia, no atribuibles a alguna otra causa. Establecer la diferencia entre estas alteraciones hipertensivas, es de importancia para el pronóstico y el tratamiento (Noriega, Arias, \& García, 2005)

La preeclampsia es una enfermedad multisistémica, de causa desconocida, propia de la mujer embarazada. Se caracteriza por una placentación anómala, con hipoxia/isquemia placentaria, disfunción del endotelio materno, probablemente favorecida por una predisposición inmunogenética con una inapropiada o exagerada respuesta inflamatoria sistémica. La enfermedad se caracteriza por la disfunción endotelial de todo el sistema materno y del lecho placentario, debido a un disbalance de los factores que promueven la normal angiogénesis a favor de factores antiangiogénicos (Direccion nacional de maternidad e infancia , 2015)

El riesgo relativo de desarrollo de preeclampsia en el embarazo actual se relaciona con: la presencia de anticuerpos antifosfolipídicos, preeclampsia en embarazo anterior, Diabetes Tipo 1 


\section{Factores causales de hipertensión arterial en mujeres durante la gestación}

Vol. 2, núm. 2., (2018)

Johanna Georgina Vidal Plúas; Walter Alejandro Patiño Zambrano; Luisana Belen Solorzano López; Steven Javier Berruz Alvarado

y 2, embarazo múltiple, historia familiar de preeclampsia en madre o hermanas, edad materna mayor a 40 años, nuliparidad e índice de masa corporal mayor a 35 . El riesgo de preeclampsia se vio también incrementado en mujeres con hipertensión crónica, enfermedad renal, enfermedades autoinmunes crónicas y con intervalo ínter genésico mayor a 10 años. (Direccion nacional de maternidad e infancia, 2015).

\section{Materiales y métodos.}

Para esta investigación se utilizó el método de la investigación documental, el cual se basa en la recopilación de documentos, seleccionarlos, analizarlos; para después mostrar resultados coherentes. Se puede puntualizar de la siguiente manera: realiza un proceso de abstracción científica, generalizando sobre la base de lo fundamental; utiliza los procedimientos lógicos y mentales de cualquier investigación; tales como: análisis, síntesis, deducción, inducción, entre otros; y, por último, supone una recopilación adecuada de datos, que permiten redescubrir hechos, sugerir problemas, ubicar hacia otras fuentes de investigación, orientar formas para elaborar instrumentos de investigación y elaborar hipótesis (Rodríguez, 2013).

Asimismo, se respaldó el trabajo con una investigación bibliográfica permite, entre otras cosas, evitar emprender investigaciones anteriormente ejecutadas, obtener conocimiento de experimentos previos para repetirlos en caso de ser necesario, buscar información sugerente, culminar investigaciones interrumpidas o incompletas, seleccionar los materiales y documentos para un marco teórico, entre otros propósitos (Rodríguez, 2013).

Con los conceptos expuestos se puede decir que en el presente estudio se utilizó como metodología una revisión bibliográfica documental no experimental, puesto que realizó una 


\section{Factores causales de hipertensión arterial en mujeres durante la gestación}

Vol. 2, núm. 2., (2018)

Johanna Georgina Vidal Plúas; Walter Alejandro Patiño Zambrano; Luisana Belen Solorzano López; Steven Javier Berruz Alvarado

revisión y análisis de diferentes artículos médicos y de opinión referentes a los factores causales de hipertensión arterial en mujeres durante la gestación, de esta manera se pudo abordar sus causas, tratamiento y consecuencias; además de poder aportar recomendaciones.

\section{Resultados.}

En pacientes con hipertensión arterial crónica se ha encontrado un aumento del riesgo de mortalidad perinatal, preeclampsia sobreagregada, parto prematuro, $\mathrm{RN}$ pequeño para la edad gestacional y cesárea. Si bien en este trabajo un importante porcentaje de pacientes presentaron preeclampsia-eclampsia sobreagregada, incluso las pacientes con hipertensión arterial crónica sin preeclampsia sobreagregada tuvieron un riesgo significativamente mayor de muerte perinatal y recién nacido pequeño para la edad gestacional que las pacientes normotensas. (Sosa \& Guirado, 2013).

La actitud inicial dependerá del tiempo de gestación y de la gravedad del proceso hipertensivo. Si el embarazo ha cumplido las 36-37 semanas y los indicadores de madurez fetal son correctos, puede adelantarse el parto, lo que pone fin a todos los problemas. Un estudio multicéntrico reciente ha demostrado que, en mujeres con HTA gestacional o PCP ligera y un embarazo a término, debería aconsejarse la inducción inmediata del parto, ya que se asocia con un menor riesgo de complicaciones fetales y maternas; como la HTA grave y el síndrome HELLP (Marin, Gorostidiz, \& Álvarez-Navascués, 2011)

La obesidad se situó en primer lugar de riesgo de desarrollar hipertensión durante el embarazo seguida de la enfermedad renal. Aunque la relación entre la obesidad y la HIE es discutida por algunos autores, para otros esta incidencia aumenta aparejada de la severidad de la 
Factores causales de hipertensión arterial en mujeres durante la gestación

Vol. 2, núm. 2., (2018)

Johanna Georgina Vidal Plúas; Walter Alejandro Patiño Zambrano; Luisana Belen Solorzano López; Steven Javier Berruz Alvarado

hipertensión. Además, la presencia de embarazos gemelares contribuye a la aparición de la HIE. (Lugo \& Álvarez, 2002)

\section{Conclusiones.}

Las mujeres que han presentado HTA durante el embarazo deben recibir consejos relacionados con hábitos de vida saludables (ejercicio físico, prevención del sobrepeso, supresión del tabaco, entre otros), además de programar con sus médicos de familia revisiones, al menos cada dos-tres años, para prevenir la aparición de HTA y la enfermedad vascular asociada. (Marin, Gorostidiz, \& Álvarez-Navascués, 2011).

La preeclampsia es la primera causa de muerte materna en el mundo desarrollado. Sólo su diagnóstico precoz permitirá instaurar un tratamiento oportuno para lograr minimizar el alto riesgo de morbimortalidad, tanto materno como perinatal. (Voto, 2008)

Es importante distinguir entre las diferentes alteraciones hipertensivas en el embarazo. Generalmente el pronóstico, tanto de la madre, como del feto es bueno, en aquellas mujeres que presentan hipertensión crónica leve o hipertensión gestacional. El tratamiento farmacológico antihipertensivo permite a las mujeres llevar su embarazo a término. Los puntos más importantes en el manejo de la preeclampsia lo constituyen el monitoreo cercano de la madre y el feto y la inducción temprana del parto. (Noriega, Arias, \& García, 2005).

\section{Recomendaciones.}




\section{Factores causales de hipertensión arterial en mujeres durante la gestación}

Vol. 2, núm. 2., (2018)

Johanna Georgina Vidal Plúas; Walter Alejandro Patiño Zambrano; Luisana Belen Solorzano López; Steven Javier Berruz Alvarado

Se recomienda el monitoreo estricto de presión arterial durante la consulta prenatal para así poder detectar a tiempo cualquiera de las patologías antes descritas y de esa forma poder controlar y evitar sus consecuencias.

Para evitar mayores complicaciones se debe actuar tratando de modificar el curso de la enfermedad ya instaurada, o vigilando estrictamente a las pacientes con factores de riesgo. Las modificaciones en el estilo de vida, incluyendo el reposo y la disminución del stress, la restricción de la ingesta diaria de sodio en las pacientes con HTA crónica, y la indicación de ácido acetilsalicílico, son intervenciones que han demostrado ser eficaces (Voto, 2008)

Las mujeres que han presentado preeclampsia o hipertensión arterial gestacional tienen, tras la menopausia, un riesgo aumentado e independiente de presentar hipertensión arterial esencial, morbimortalidad cardiovascular y enfermedad renal terminal. Por lo cual necesitan un seguimiento selectivo por parte de sus médicos de familia (Marin, Gorostidiz, \& ÁlvarezNavascués, 2011).

\section{Bibliografía.}

Direccion nacional de maternidad e infancia . (2015). Guía para el diagnostico y trataiento de la hipertension en el embarazo. . Guía para el diagnostico y trataiento de la hipertension en el embarazo. .

Lugo, A., \& Álvarez, V. y. (2002). Factores epidemiológicos de la hipertensión en el embarazo. Toko Gin. Pract., 317-320.

Marin, R., Gorostidiz, M., \& Álvarez-Navascués, R. (2011). Hipertensión arterial y embarazo. NefroPlus, 21-29.

Noriega, M., Arias, E., \& García, S. (2005). Hipertensión arterial en el embarazo. Médica Sur, 196-202. 


\section{Factores causales de hipertensión arterial en mujeres durante la gestación}

Vol. 2, núm. 2., (2018)

Johanna Georgina Vidal Plúas; Walter Alejandro Patiño Zambrano; Luisana Belen Solorzano López; Steven Javier Berruz Alvarado

Rodríguez, M. (2013). Acerca de la investigación bibliográfica y documental. Obtenido de Guía de tesis: http://guiadetesis. wordpress. com/2013/08/19/acerca-de-la-investigacionbibliografica-ydocumental

Sosa, L., \& Guirado, M. (2013). Estados hipertensivos en el embarazo . Revista Uruguaya de Cardiología, 285-295.

Voto, L. (2008). Hipertension en el embarazo. Seccion hipertension arterial. Hipertensión en el embarazo, 50-62. 